\title{
GLASS TRANSITION AT INTERFACES
}

- Thomas Salez ${ }^{1,2,3}$, Joshua D. McGraw ${ }^{4,5}$, Kari Dalnoki-Veress ${ }^{1,5}$, Elie Raphaël', James A. Forrest ${ }^{1,2,6}$

I 1 Laboratoire de Physico-Chimie Théorique, UMR CNRS Gulliver 7083, ESPCI Paris, PSL Research University, 75005 Paris, France.

$1{ }^{2}$ Perimeter Institute for Theoretical Physics, Waterloo, ON N2L 2Y5, Canada.

${ }^{3}$ Global Station for Soft Matter, Global Institution for Collaborative Research and Education, Hokkaido University, Sapporo, Hokkaido 060-0808, Japan.

I ${ }^{4}$ Laboratoire de Physique Statistique, Ecole Normale Supérieure, 75005 Paris, France.

- ${ }^{5}$ Department of Physics and Astronomy, McMaster University, Hamilton, ON L8S 4M1, Canada.

- ${ }^{6}$ Department of Physics \& Astronomy, University of Waterloo, Waterloo, ON N2L 3G1, Canada.

I DOI: https://doi.org/10.1051/epn/2017103

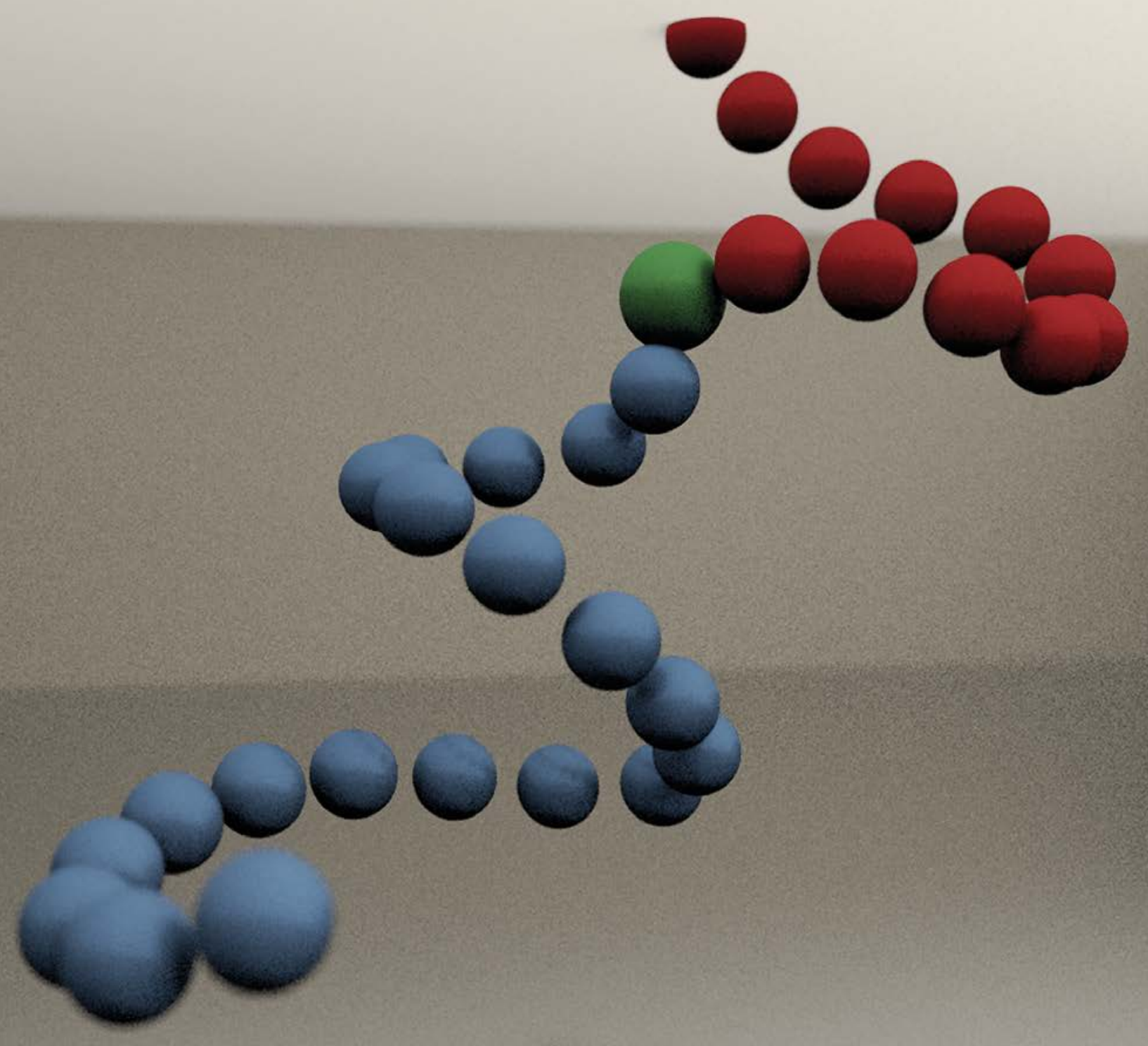




\section{We live in the twenty-first century. Almost all the changes between phases of matter are described by the theory of phase transitions. Yet, there is one phenomenon that still resists theoretical attempts at its description: the glass transition. Moreover, signatures of the glass transition seem to be modified in thin polymer films. These observations may provide insights into the detailed mechanisms of the transition and guide the use of thin films through promising applications. It thus appears crucial to understand the effects of confinement and interfaces on the glass transition.}

\begin{abstract}
A mysterious transition
The glass transition is a change of state between a viscous liquid and an amorphous solid obtained by cooling. What are the mechanisms at play in this phenomenon when the atomic structures themselves seem unchanged? Why is this common physical fact, mastered by glass blowers since the Roman Empire, so difficult to understand and why does it appear so different from the classical liquid-solid transition? These questions are far from being new but continue to stimulate important research activities [1]. This large effort is due to the central role of glasses and plastics (amorphous solids made from polymers) in art, industry and nanotechnology, as well as their broad diversity, which ranges from molecular to organic and polymer glasses, including metallic, spin and colloidal glasses, as well as jammed granular media.
\end{abstract}

\section{Cooperativity and confinement}

Despite the lack of a unified theory, a central concept appeared in the sixties from the work of Adam and Gibbs,in order to describe glassy dynamics: cooperativity. In usual liquids, molecular motions are uncorrelated. By contrast, crowding within glasses is important, and the dynamics might rely on spatial regions of size $\xi$ in which the molecules rearrange collectively. This cooperative length scale, expected to grow rapidly near some finite temperature, could be the ideal observable for the glass transition, and continues to be the focus of intense investigations. Unfortunately, it is impractical to wait for macroscopic structural rearrangements in a glass due to the enormous time scales involved compared to those accessible to experiment. Furthermore, it seems unrealistic to resolve individual molecular trajectories in the bulk with existing microscopy techniques. One thus cannot currently measure $\xi$ in a bulk glass.

An alternative strategy emerged in the seventies: making glassy systems of a size comparable to $\xi$ and observing their anomalies. However, since the elementary unit involved in $\xi$ is the molecular diameter, one had to prepare and dynamically observe stable samples of a few molecular diameters. This was an impossible task, $a$ priori. Polymer films offered an exception for a couple of reasons: first, polymer chains have a remarkable stability in the liquid phase (as noticed by industrialists); secondly, the sample preparation is relatively easy.

\section{Anomalies in polymer nanofilms}

Like most materials, a polymer film expands with temperature. This dilatation can be detected and precisely quantified by techniques such as ellipsometry (a measure of the change in the polarisation of light after reflection on a sample). By lowering the temperature, the system progressively contracts with a constant thermal expansion coefficient. Then, at a given temperature (about $100^{\circ} \mathrm{C}$ for the case of polystyrene) the expansion coefficient changes abruptly: the point of this observed transition, and a related measure based on calorimetry, is often defined as the glass-transition temperature $T_{g}$.

In the nineties, using these methods, several teams reported that the glass-transition temperature of a polystyrene film depends on its average thickness, when the thickness is below $50 \mathrm{~nm}$ typically (Fig. 1, circles). In addition, experiments focusing on local dynamics, realized through embedding of gold nanoparticles, recording of capillary fluctuations, or tracer evolution, revealed a second anomaly in polymer films: the existence of a liquid-like mobile layer localized over a few nanometers

v FIG. 1:Problem. Glass-transition temperature $T_{g}$ of polystyrene films supported on silicon substrates, measured by ellipsometry, as a function of film thickness $h$ (closed circles). For comparison, the literature data is indicated (open circles). The microscopic hypothesis of the random cooperative strings (see end of the article) allows one to quantify the local molecular mobility and thus the glass-transition temperature (solid line).

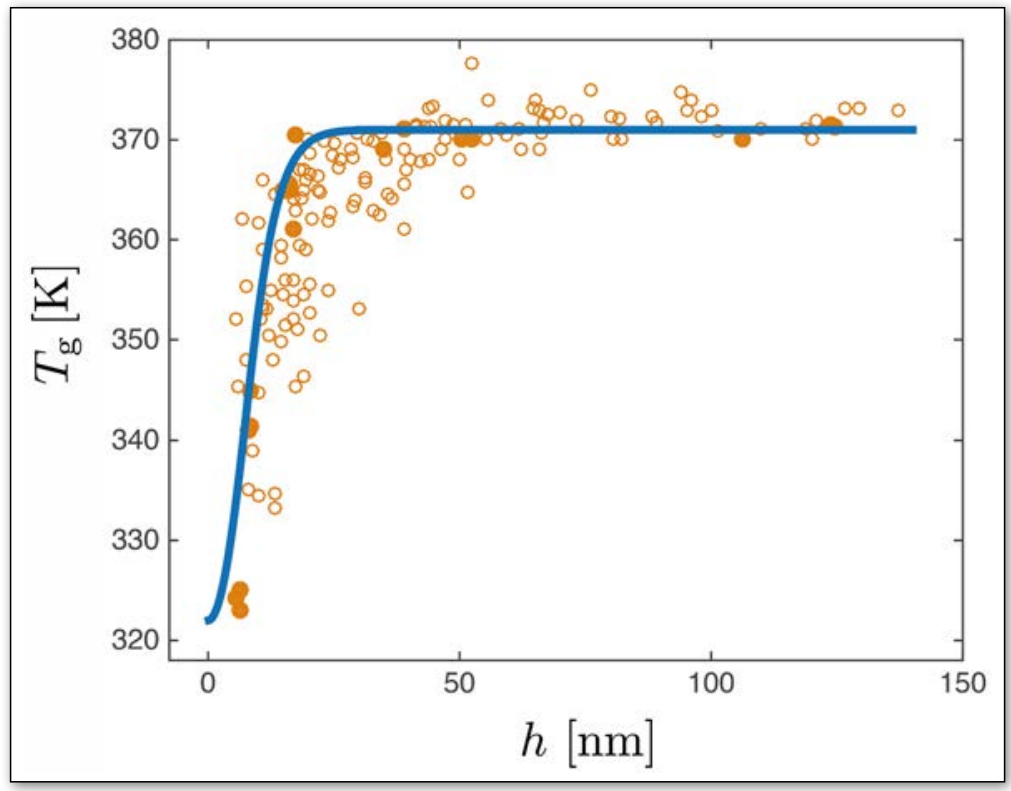




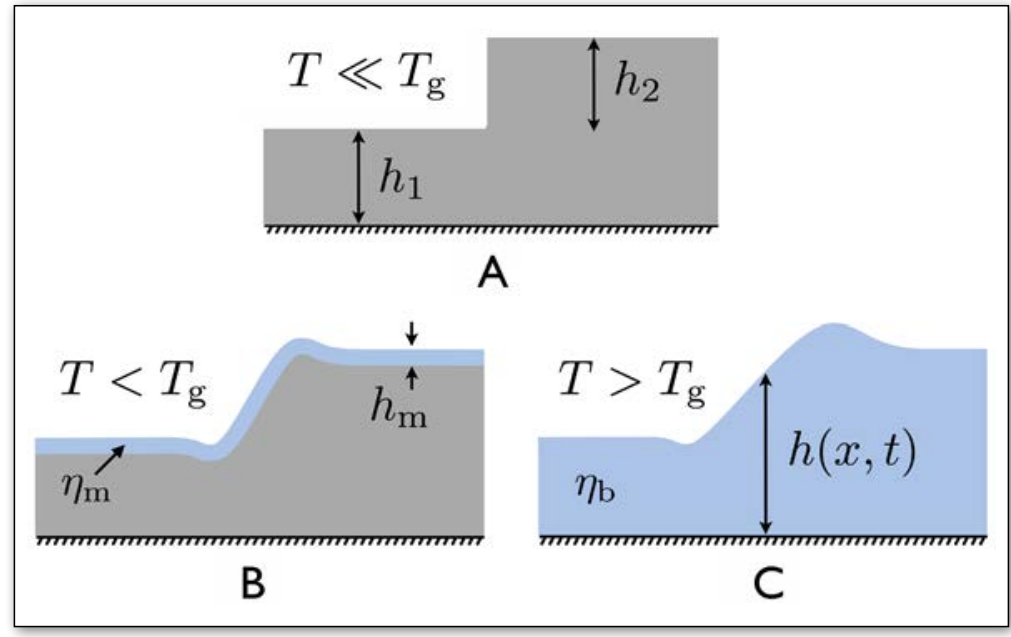

$\triangle$ FIG.2: System and notations. As prepared, the sample is a polystyrene stepped film with thicknesses $h_{1}$ and $h_{2}$ on the order of $100 \mathrm{~nm}$, at room temperature $T$ well below the glass-transition temperature $T_{g}$.

near the free surface. The interpretation of these measurements is still hotly contested. The origin and properties of the mobile layer are undetermined, and the link with the $T_{g}$ shift remains hypothetical.

\section{Interest in thin films and their rheology}

Microfilms and nanofilms of polymers - molten, rubbery, or glassy - are of tremendous interest in a broad variety of interdisciplinary fields, such as physical chemistry, physiology, biophysics, microelectronics or surface science. Besides their fundamental importance, such films are frequently involved in industrial processes, optical mechanical or chemical, through nanolithography, lubrication, paints, surface treatments, and elastic membranes. Future development of molecular electronics, organic multimedia displays, biomimetic devices, superadhesive or self-cleaning surfaces may even increase

v FIG. 3: Observations. (A) Atomic-force-microscopy images of the levelling of a polystyrene (PS) stepped film, with $15 \mathrm{~kg} / \mathrm{mol}$ molecular weight, initially and after some time $t$. The capillary stresses in the corners of the step are such that Laplace pressure generates a viscous flow together with surface oscillations and relaxation. (B) Comparison between experimental profile $h(x, t)$, where $x$ is the horizontal position, and hydrodynamic model, for two different geometries (top: thick step, bottom: thin step), at time $t=10 \mathrm{~min}$. The only adjustable parameter is an overall horizontal stretch factor related to the film viscosity $\eta_{b}$, and thus allowing for the latter's precise measurement.
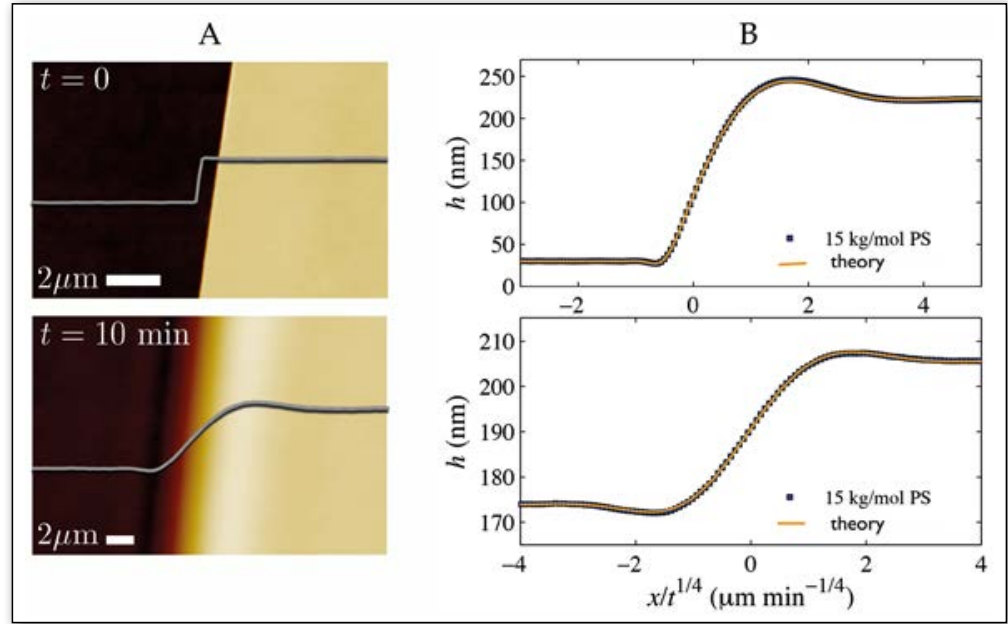

the importance of such materials. As an example, thin polymer films are potential candidates for massive data storage, through the IBM Millipede project. The idea is to indent the film surface at the nanoscale, in order to mechanically imprint information, and by this means to increase considerably the surface storage density with respect to microelectronic standards.

Through these potential applications, one easily recognizes that a detailed understanding of the physics at play behind the stability and dynamics of these systems is necessary. When the average thickness (10-100 nm) of polymer nanofilms is comparable to the typical macromolecular size, the effects of confinement may drastically alter their properties. The nanorheology of these systems raises a number of questions that are currently highly debated, at the boundary between the continuous and discrete descriptions of matter. Besides the glass-transition anomalies - central to the present article - let us provide two examples. First, the modification of intramolecular conformations and intermolecular entanglements, near surfaces and in confined settings, might directly impact the viscosity and thus the stability time scale of thin films. Secondly, interfacial phenomena may dominate over volume effects in thin geometries, and thus the overall rheology might strongly depend on the interfacial frictional and slip properties - the latter of which is strongly amplified for entangled polymers.

\section{The stepped-film technique}

In order to understand the glass-transition anomalies in thin films, and more generally their rheology, a nanoprobe is needed. Such a probe is a device or method enabling the measurement of physical quantities at the nanoscale. For instance, in order to know if a material flows and to quantify this flow, the free interface of the sample can be deformed and the resulting evolution observed. This is what we instinctively realize when perturbing the surface of honey in a pot with a spoon: the deformation progressively vanishes under the action of gravity. That relaxation operates over a time scale that increases as the honey is cooled, which is directly related to the viscosity of the material.

At the nanoscale, the dominant driving force is surface tension (which also controls the shape of small water droplets and soap bubbles) rather than gravity. By starting with a non-flat polymer nanofilm supported on a rigid substrate, and by observing its capillary levelling over time, one can thus deduce the internal mobility of the material [2]. More precisely, the developed technique consists of making a polystyrene stepped film (Fig. 2) - by superimposing two flat films with typical thicknesses on the order of several tens of nanometers but with different horizontal extents - and then observing its relaxation using atomic force microscopy (Fig. 3A). The evolution and shape of the profile being well described by fluid 
mechanics, one can precisely calibrate this nanoprobe (Fig. 3B).

\section{Measure of surface mobility in a glass}

Using the calibrated stepped-film technique, it is then possible to study the capillary relaxation of polystyrene films below their glass-transition temperature $T_{g}$ [3]. The conclusion is striking: the surface evolves! The evolution occurs over several hours, and is even observed a few tens of degrees below $T_{g}$. These observations can be understood using a simple two-layer mesoscopic model. Below the glass-transition temperature, the dynamics are heterogeneous and the film exhibits a liquid-like mobile layer (Fig. 4A) whose thickness increases and reaches the whole sample size (Fig. 4B) at the glass transition. The important result is that the mobility of this surface layer can be extracted (Fig. 5) from a fit to experimental data (Figs. 3B, 4A, and 4B).

\section{The cooperative-string model}

In order to understand the physical origin of such a liquid-like surface layer atop glassy films, and its possible link with the observed $T_{g}$ shifts, one needs to develop a microscopic theory [4]. Inside a bulk glass, molecules are in an environment similar to the subway at rush hour. Each molecule is trapped in a cage formed by its nearest neighbours, and has very little free volume to move, to change position, and thus to allow for the material to relax towards equilibrium. Given the impossibility of individual motion, an alternative mechanism becomes cooperative motion: several molecules move simultaneously, just as passengers during the rearrangements occurring at stations. Moreover, using observations made in colloids, granular media and numerical simulations, one can assume that the cooperatively rearranging regions have the shape of one-dimensional chains of molecules rather than three-dimensional clusters. These are the socalled random cooperative strings. Based on this idea, and thanks to statistical physics, it is then possible to recover the usual phenomenology of bulk glasses.

Finally, one can add a free interface to the description above. Doing so, it is possible to address quantitatively the two anomalies introduced previously. The key ingredient lies in the fact that a random cooperative string is truncated at the free interface due to the absence of crowding constraints (Fig. 4C). In other words, a passenger of the subway standing close to the exit gate does not require any collective motion to escape the train: the passenger can simply jump outside, as a liquid-like molecule. This type of behaviour fluidizes the neighbourhood of the gate. Invoking a Brownian description for the cooperative chains and the first-passage density of probability at the interface, one can quantify the local relaxation time in a thin film, and thus its mobility and glass-transition properties (Fig. 1).

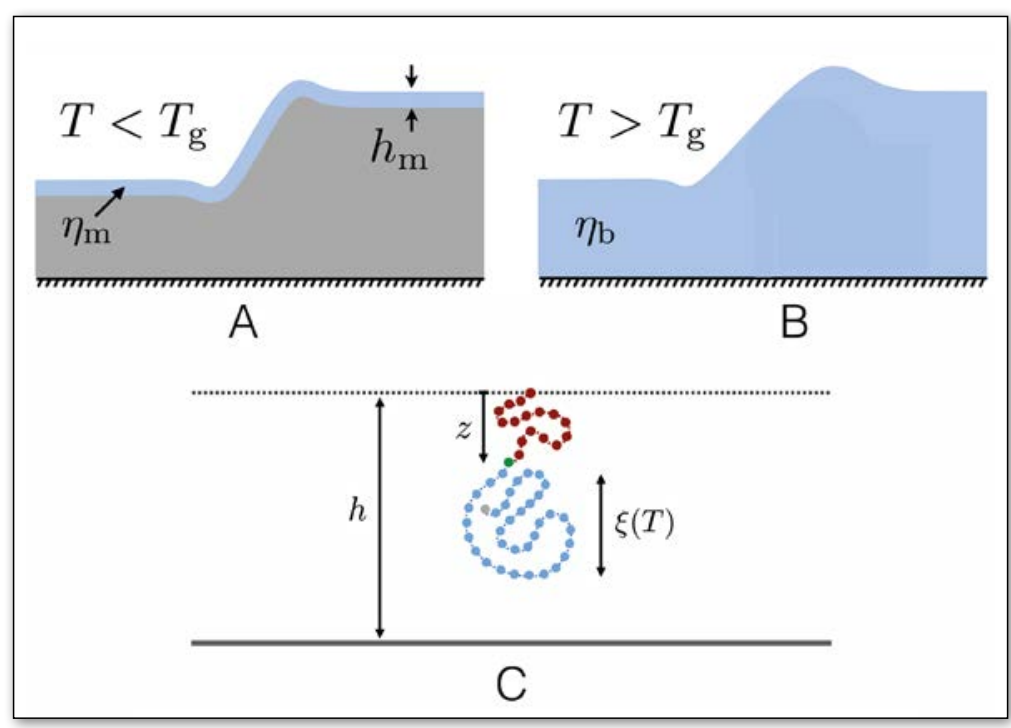

$\triangle$ FIG.4: Models. (A) (mesoscopic) For a temperature range below $T_{g}$, the flow is localized in a surface layer (blue) of thickness $\boldsymbol{h}_{m}$, on the order of a few nanometers. As the surface evolves through the flow of the blue region, an initially immobile region (grey) can reach a distance to the free surface that is smaller than $\boldsymbol{h}_{m}$, and thus becomes liquid (blue). This explains the rearrangement of the grey region as well.(B) (mesoscopic) For a temperature range above $T_{g}$, the flow occurs in the whole sample. (C) (microscopic) Truncation of cooperative strings at the free interface of a glassy film. At a temperature $T$, in a film of thickness $h$, and at a distance $z$ from the free interface (dashed line), a molecule (green) can relax through a random cooperative string (blue) of size $\xi$ in the bulk of the sample, but it can alternatively do so through a string that is truncated by the interface (red) - shorter and thus more probable - which increases the surface mobility and thus reduces the effective glass-transition temperature of the film.

\section{In order to know if a material flows and to quantify this flow, the free interface of the sample can be deformed and the resulting evolution observed.}

v FIG. 5: Results. Poiseuille mobility $H^{3} /(3 \eta)$ of a polymer film (defined by the thickness $H$ and viscosity $\eta$ of the flow region) as a function of the inverse temperature $1 / T$. As a characteristic feature of the glass transition, the mobility varies over ten decades near $T_{g}$. When $T>T_{g}$ (squares), the flow occurs within the whole film (Fig.4B), with an average thickness $H=h_{1}+h_{2} / 2$ and bulk viscosity $\eta=\eta_{b}$. The viscosity satisfies the expected Vogel-Fulcher-Tammann (VFT) law for supercooled liquids. When $T<T_{g}$ (circles), the flow is localized in a near-surface region (Fig. 4A) of thickness $H=h_{m}$, and viscosity $\eta=\eta_{m}$ which seems to follow an Arrhenius-like law, reminiscent of simple liquids. The viscosity $\eta_{m}$ and thickness $h_{m}$ of the mobile layer cannot be determined independently in such a measurement.

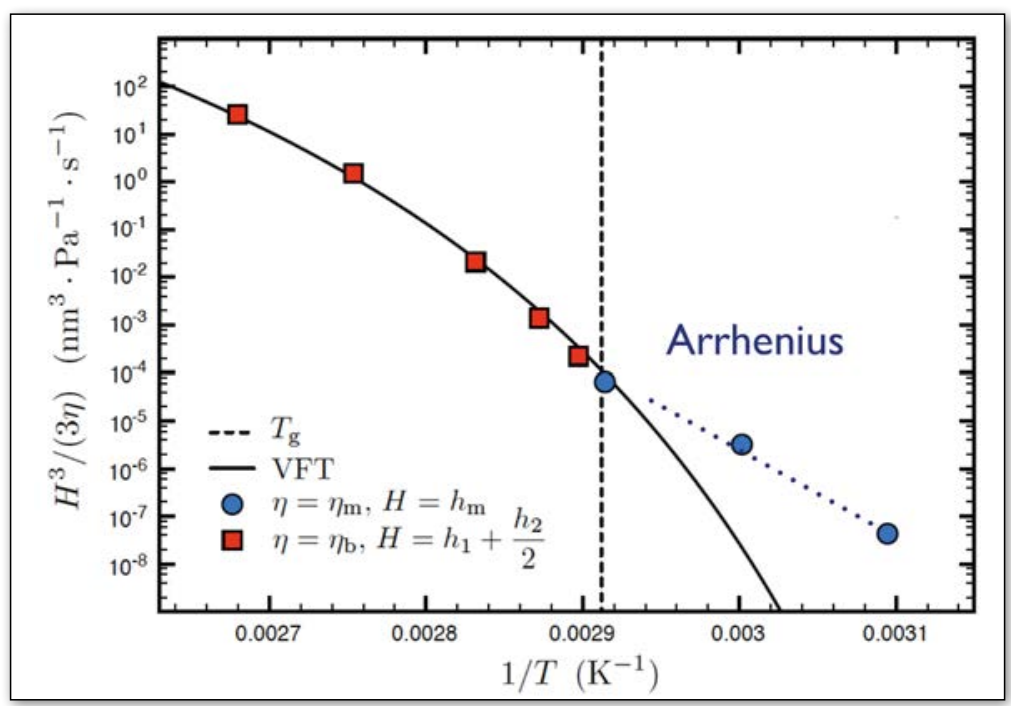




\section{Conclusion}

It is often thought that windows in cathedrals are thicker at the bottom because the glass slowly flows. Ironically, this hypothesis, while wrong for the windows, appears to be verified in amorphous nanofilms, near their glass-transition temperature. For instance, a polymer glass - a fundamental material in industry and nanotechnology - is traditionally described as a disordered solid, but it flows! This flow occurs near the free surface, and over a few nanometers in depth, which reduces the effective glass-transition temperature.

The stepped-film technique facilitates the ability to characterize the dynamic properties of glassy polymeric nanolayers as a function of temperature and for various compositions. The method is not limited to those materials and could more generally allow one to probe soft condensed matter in confinement, at the nanoscale, where interfaces and finite molecular sizes play a dominant role.
Finally, the microscopic cooperative-string theory presents two advantages: its analytical simplicity and its pictorial character, which might contribute to make it a practical tool to study the glass transition. One can thus hope to better understand the mechanisms of this phenomenon, as well as its anomalies in thin films and nanoparticles [5], and, for instance, to guide the fabrication of ultrastable glasses, these fascinating emerging materials.

\section{Acknowledgments}

These results were obtained within an international and interdisciplinary collaborative network, including:Yu Chai (Waterloo University, Canada), Justin Salez (Université Paris Diderot, France), Oliver Bäumchen (Max Planck Institute, Göttingen, Germany), Michael Benzaquen (CFM, Paris, France), and Maxence Arutkin (ESPCI Paris, France). 


\section{About the authors}

Thomas Salez is a CNRS research associate at ESPCI Paris, France, and an assistant professor at Hokkaido University, Sapporo, Japan.

Joshua D. McGraw is a junior research chair in the department of physics at École Normale Supérieure, Paris, France.

Kari Dalnoki-Veress is a professor in the department of physics and astronomy at McMaster University, Hamilton, Ontario, Canada.

Elie Raphaël is a CNRS research director at ESPCI Paris, France.

James A. Forrest is a professor in the department of physics and astronomy at the University of Waterloo, and the academic program director at the Perimeter Institute for Theoretical Physics, Waterloo, Ontario, Canada.

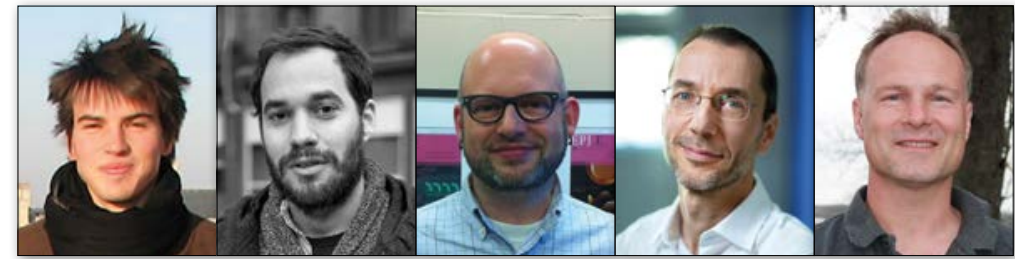

$\Delta$ Left to right:

Thomas Salez,

Joshua McGraw,

Kari Dalnoki-Veress,

Elie Raphaël

and James Forrest

\section{References}

[1] L. Berthier and M. D. Ediger, Physics Today 69, 40 (2016).

[2] J. D. McGraw, T. Salez, O. Bäumchen, E. Raphaël, and K. Dalnoki-Veress, Physical Review Letters 109, 128303 (2012).

[3] Y. Chai, T. Salez, J. D. McGraw, M. Benzaquen, K. Dalnoki-Veress, E. Raphaël, and J. A. Forrest, Science 343, 994 (2014).

[4] T. Salez, J. Salez, K. Dalnoki-Veress, E. Raphaël, and J. A. Forrest, Proceedings of the National Academy of Sciences of the USA 112, 8227 (2015).

[5] M. Arutkin, E. Raphaël, J. A. Forrest, T. Salez, Soft Matter 13, 141 (2017). 